\title{
Asymptotic Estimates for Second-Order Parameterized Singularly Perturbed Problem
}

\author{
Mustafa Kudu \\ Department of Mathematics, Faculty of Art and Science, Erzincan University, Erzincan, Turkey \\ Email: muskud28@yahoo.com \\ Received 16 April 2014; revised 20 May 2014; accepted 3 June 2014 \\ Copyright (C) 2014 by author and Scientific Research Publishing Inc. \\ This work is licensed under the Creative Commons Attribution International License (CC BY). \\ http://creativecommons.org/licenses/by/4.0/

c. (i) Open Access

\section{Abstract}

The boundary value problem (BVP) for parameterized singularly perturbed second order nonlinear ordinary differential equation is considered. The boundary layer behavior of the solution and its first and second derivatives have been established. An example supporting the theoretical analysis is presented.

\section{Keywords}

Parameterized Problem, Asymptotic Bounds, Singular Perturbation, Boundary Layer

\section{Introduction}

In this paper, we are going to obtain the asymptotic bounds for the following parameterized singularly perturbed boundary value problem (BVP):

$$
\begin{gathered}
L u:=\varepsilon u^{\prime \prime}(t)+a(t) u^{\prime}(t)-f(t, u(t), \lambda)=0, \quad 0<t<T, \\
u(0)=\mu_{0}, \quad u^{\prime}(0)=\frac{\mu_{1}}{\varepsilon}, u(T)=\mu_{2},
\end{gathered}
$$

where $0<\varepsilon \leq 1$ is a perturbation parameter, $\mu_{i}(i=0,1,2)$ are given constants and $0<\alpha \leq a(t) \leq a^{*}$ is a sufficiently smooth function in $[0, T]$. Further, the function $f(t, u(t), \lambda)$ is assumed to be sufficiently continuously differentiable for our purpose function in $\{0 \leq t \leq T,-\infty<u<\infty,-\infty<\lambda<\infty\}$ and

$$
0 \leq \frac{\partial f}{\partial u} \leq M_{i}^{*}, \quad 0<m_{1} \leq\left|\frac{\partial f}{\partial \lambda}\right| \leq M_{1}<\infty .
$$


By a solution of (1), (2) we mean pair $\{u(t), \lambda\} \in C^{1}[0, T] \times \mathbb{R}$ for which problem (1), (2) is satisfied.

An overview of some existence and uniqueness results and applications of parameterized equations may be obtained, for example, in [1]-[10]. In [11]-[14], some approximating aspects of this kind of problems have also been considered. The qualitative analysis of singular perturbation situations have always been far from trivial because of the boundary layer behavior of the solution. In singular perturbation cases, problems depend on a small parameter $\varepsilon$ in such a way that the solution exhibits a multiscale character, i.e., there are thin transition layers where the solution varies rapidly while away from layers it behaves regularly and varies slowly [15] [16]. In this note we establish the boundary layer behaviour for $u(t)$ of the solution of (1)-(2) and its first and second derivatives. Example that agree with the analytical results is given.

Theorem 1.1. For $\rho=1-\alpha^{-1} m_{1}^{-1} M_{1}^{-1} T>0$ the solution $\{u(t), \lambda\}$ of the problem (1), (2) satisfies,

$$
\begin{aligned}
& |\lambda| \leq c_{0}, \\
& |u| \leq c_{1},
\end{aligned}
$$

where

$$
\begin{gathered}
c_{0}=\rho^{-1}\left[\frac{m_{1} a^{*}}{T-\left(a^{*}\right)^{-1}\left(1-\mathrm{e}^{-a^{*} T}\right)}\left(\left|\mu_{0}\right|+\left|\mu_{2}\right|+\alpha^{-1} T\|F\|_{\infty}+\alpha^{-1} M_{1} T\right)\right], \\
c_{1}=\left|\mu_{0}\right|+\left|\mu_{2}\right|+\alpha^{-1} T\|F\|_{\infty}+\alpha^{-1} c_{0} M_{1} T, \quad F(t)=f(t, 0,0)
\end{gathered}
$$

and

$$
\left|u^{(k)}(t)\right| \leq C\left(1+\frac{1}{\varepsilon^{k}} \mathrm{e}^{-\frac{\alpha t}{\varepsilon}}\right), k=1,2, t \in[0, T]
$$

provided $a \in C^{1}[0, T]$ and $\left|\frac{\partial f}{\partial t}\right| \leq C$ for $t \in[0, T]$ and $|u| \leq c_{1}, \quad|\lambda| \leq c_{0}$.

Proof. We rewrite Equation (1) in the form

$$
\varepsilon u^{\prime \prime}(t)+a(t) u^{\prime}(t)-b(t) u(t)-\lambda c(t)-F(t)=0, t \in[0, T],
$$

where,

$$
b(t)=\frac{\partial f}{\partial u}(t, \tilde{u}, \tilde{\lambda}), c(t)=\frac{\partial f}{\partial \lambda}(t, \tilde{u}, \tilde{\lambda}), \tilde{u}=\gamma u, \tilde{\lambda}=\gamma \lambda \quad(0<\gamma<1) \text {-intermediate values. }
$$

From (7) for the first derivate, we have

$$
\begin{aligned}
u^{\prime}(t)= & \frac{\mu_{1}}{\varepsilon} \mathrm{e}^{-\frac{1}{\varepsilon} \int a(\tau) \mathrm{d} \tau}+\frac{1}{\varepsilon} \int_{0}^{t} b(\tau) u(\tau) \mathrm{e}^{-\frac{1}{\varepsilon} \delta a(\eta) \mathrm{d} \eta} \mathrm{d} \tau+\frac{\lambda}{\varepsilon} \int_{0}^{t} c(\tau) \mathrm{e}^{-\frac{1}{\varepsilon} \int a(\eta) \mathrm{d} \eta} \mathrm{d} \tau \\
& +\frac{1}{\varepsilon} \int_{0}^{t} F(\tau) \mathrm{e}^{-\frac{1}{\varepsilon} \varepsilon_{\tau}^{t} a(\eta) \mathrm{d} \eta} \mathrm{d} \tau .
\end{aligned}
$$

Integrating this equality over $(0, t)$ we get

$$
\begin{aligned}
u(t)= & \mu_{0}+\frac{\mu_{1}}{\varepsilon} \int_{0}^{t} \mathrm{e}^{-\frac{1}{\varepsilon} \int_{0}^{\xi} a(\tau) \mathrm{d} \tau} \mathrm{d} \xi+\frac{1}{\varepsilon} \int_{0}^{t} \mathrm{~d} \tau b(\tau) u(\tau) \int_{\tau}^{t} \mathrm{e}^{-\frac{1}{\varepsilon} \int_{\tau}^{\xi} a(\eta) \mathrm{d} \eta} \mathrm{d} \xi \\
& +\frac{1}{\varepsilon} \int_{0}^{t} \mathrm{~d} \tau F(\tau) \int_{\tau}^{t} \mathrm{e}^{-\frac{1}{\varepsilon} \int_{\tau}^{\xi} a(\eta) \mathrm{d} \eta} \mathrm{d} \xi+\frac{\lambda}{\varepsilon} \int_{0}^{t} \mathrm{~d} \tau c(\tau) \int_{\tau}^{t} \mathrm{e}^{-\frac{1}{\varepsilon} \int_{\tau}^{\xi} a(\eta) \mathrm{d} \eta} \mathrm{d} \xi
\end{aligned}
$$

from which by setting the boundary condition $u(T)=\mu_{2}$, we obtain, 
M. Kudu

$$
\begin{aligned}
& \lambda=\frac{\varepsilon\left(\mu_{0}-\mu_{2}\right)}{\int_{0}^{T} \mathrm{~d} \tau c(\tau) \int_{\tau}^{T} \mathrm{e}^{-\frac{1}{\varepsilon} \int_{\tau}^{\xi} a(\eta) \mathrm{d} \eta} \mathrm{d} \xi}+\frac{\int_{0}^{T} \mathrm{e}^{-\frac{1}{\varepsilon} \int_{0}^{\xi} a(\tau) \mathrm{d} \tau} \mathrm{d} \xi}{\int_{0}^{T} \mathrm{~d} \tau c(\tau) \int_{\tau}^{T} \mathrm{e}^{-\frac{1}{\varepsilon_{\tau}} \int_{\tau} a(\eta) \mathrm{d} \eta} \mathrm{d} \xi}+\frac{\int_{0}^{T} \mathrm{~d} \tau b(\tau) u(\tau) \int_{\tau}^{T} \mathrm{e}^{-\frac{1}{\varepsilon} \int_{\tau}^{\xi} a(\eta) \mathrm{d} \eta} \mathrm{d} \xi}{\int_{0}^{T} \mathrm{~d} \tau c(\tau) \int_{\tau}^{T} \mathrm{e}^{-\frac{1}{\varepsilon} \int_{\tau}^{\xi} a(\eta) \mathrm{d} \eta} \mathrm{d} \xi} \\
& +\frac{\int_{0}^{t} \mathrm{~d} \tau F(\tau) \int_{\tau}^{t} \mathrm{e}^{-\frac{1}{\varepsilon} \int_{\tau}^{\xi} a(\eta) \mathrm{d} \eta} \mathrm{d} \xi}{\int_{0}^{T} \mathrm{~d} \tau c(\tau) \int_{\tau}^{T} \mathrm{e}^{-\frac{1}{\varepsilon} \int_{\tau}^{\xi} a(\eta) \mathrm{d} \eta} \mathrm{d} \xi}
\end{aligned}
$$

Applying the mean value theorem for integrals, we deduce that,

$$
\frac{\left|\int_{0}^{t} \mathrm{~d} \tau F(\tau) \int_{\tau}^{t} \mathrm{e}^{-\frac{1}{\varepsilon} \int_{\tau}^{\xi} a(\eta) \mathrm{d} \eta} \mathrm{d} \xi\right|}{\left|\int_{0}^{T} \mathrm{~d} \tau c(\tau) \int_{\tau}^{T} \mathrm{e}^{-\frac{1}{\int_{\tau}^{\xi}} a(\eta) \mathrm{d} \eta} \mathrm{d} \xi\right|} \leq \frac{\left|F \|_{\infty}\right| \int_{0}^{t} \mathrm{~d} \tau \int_{\tau}^{t} \mathrm{e}^{-\frac{1}{\varepsilon_{\tau}} \int_{\tau}^{\xi} a(\eta) \mathrm{d} \eta} \mathrm{d} \xi \mid}{m_{1}\left|\int_{0}^{T} \mathrm{~d} \tau \int_{\tau}^{T} \mathrm{e}^{-\frac{1}{\varepsilon_{\tau}} a(\eta) \mathrm{d} \eta} \mathrm{d} \xi\right|} \leq\|F\|_{\infty} m_{1}^{-1},
$$

and

$$
\frac{\left|\int_{0}^{t} \mathrm{~d} \tau b(\tau) u(\tau) \int_{\tau}^{t} \mathrm{e}^{-\frac{1}{\varepsilon} \int_{\tau}^{\xi} a(\eta) \mathrm{d} \eta} \mathrm{d} \xi\right|}{\left|\int_{0}^{T} \mathrm{~d} \tau c(\tau) \int_{\tau}^{T} \mathrm{e}^{-\frac{1}{\varepsilon} \int_{\tau}^{\xi} a(\eta) \mathrm{d} \eta} \mathrm{d} \xi\right|} \leq \frac{\left|u \|_{\infty}\right| \int_{0}^{t} \mathrm{~d} \tau b(\tau) \int_{\tau}^{t} \mathrm{e}^{-\frac{1}{\varepsilon} \int_{\tau} a(\eta) \mathrm{d} \eta} \mathrm{d} \xi \mid}{m_{1}\left|\int_{0}^{T} \mathrm{~d} \tau \int_{\tau}^{T} \mathrm{e}^{-\frac{1}{\int_{\tau}} \int_{\tau} a(\eta) \mathrm{d} \eta} \mathrm{d} \xi\right|} \leq m_{1}^{-1} M_{i}^{*}\|u\|_{\infty} .
$$

Also, for first and second terms in right side of (10), for $\varepsilon \leq 1$ values, we have

$$
\begin{aligned}
& \frac{\varepsilon\left|\mu_{0}-\mu_{2}\right|}{\left|\int_{0}^{T} \mathrm{~d} \tau c(\tau) \int_{\tau}^{T} \mathrm{e}^{-\frac{1}{\varepsilon} \int_{\tau}^{\xi} a(\eta) \mathrm{d} \eta} \mathrm{d} \xi\right|}+\frac{\left|\mu_{1}\right| \int_{0}^{T} \mathrm{e}^{-\frac{1}{\varepsilon} \int_{0}^{\xi} a(\tau) \mathrm{d} \tau} \mathrm{d} \xi}{\left|\int_{0}^{T} \mathrm{~d} \tau c(\tau) \int_{\tau}^{T} \mathrm{e}^{-\frac{1}{\varepsilon} \int_{\tau}^{\xi} a(\eta) \mathrm{d} \eta} \mathrm{d} \xi\right|} \\
& \leq \frac{m_{1}^{-1}\left(a^{*}\right)\left|\mu_{0}-\mu_{2}\right|}{T-\varepsilon\left(a^{*}\right)^{-1}\left(1-\mathrm{e}^{-\frac{a^{*} T}{\varepsilon}}\right)}+\frac{\alpha^{-1} m_{1}^{-1}\left(a^{*}\right)\left|\mu_{1}\right|}{T-\varepsilon\left(a^{*}\right)^{-1}\left(1-\mathrm{e}^{-\frac{a^{*} T}{\varepsilon}}\right)} \\
& \leq \frac{m_{1}^{-1}\left(a^{*}\right)}{T-\varepsilon\left(a^{*}\right)^{-1}\left(1-\mathrm{e}^{-\frac{a^{*} T}{\varepsilon}}\right)}\left[\left|\mu_{0}-\mu_{2}\right|+\alpha^{-1}\left|\mu_{1}\right|\right],
\end{aligned}
$$

It then follows from (11)-(13)

$$
|\lambda| \leq \frac{m_{1}^{-1}\left(a^{*}\right)}{T-\varepsilon\left(a^{*}\right)^{-1}\left(1-\mathrm{e}^{-a^{*} T}\right)}\left[\left|\mu_{0}-\mu_{2}\right|+\alpha^{-1}\left|\mu_{1}\right|\right]+m_{1}^{-1}\|F\|_{\infty}+m_{1}^{-1} M_{1}^{*}\|u\|_{\infty}
$$

Next from (9), we see that 


$$
\|u\|_{\infty} \leq\left|\mu_{0}\right|+\left|\mu_{2}\right|+\alpha^{-1} T\|F\|_{\infty}+\alpha^{-1}|\lambda| T,
$$

Under the conditions $0<\alpha \leq a(t) \leq a^{*}$ and $0 \leq b(t) \leq M_{1}^{*}$ the operator $L v:=\varepsilon v^{\prime \prime}(t)+a(t) v^{\prime}(t)-b(t) v(t)$ admits the following maximum principle: Suppose $v(t) \in C^{2}[0, T]$ be any function satisfiying $L v \leq 0(0<t<T), v(0) \geq 0$ and $v(T) \geq 0$, then $v(t) \geq 0(0<t<T)$.

Using the maximum principle whith barrier functions $\psi_{ \pm}(t)= \pm v(t)+\alpha^{-1}(T-t)|| L v \|_{\infty}+|v(0)|+|v(T)|$ we have the inequality

$$
\|u\|_{\infty} \leq\left|\mu_{0}\right|+\left|\mu_{2}\right|+\alpha^{-1} T\|F\|_{\infty}+\alpha^{-1}|\lambda| T .
$$

The inequlities (14), (15) immediately leads to (4), (5). After taking into consideration the uniformly boundnees in $\varepsilon$ of $u(t)$ and $\lambda$, it then follows from (8) that,

$$
u^{\prime}(t)=\frac{\left|\mu_{1}\right|}{\varepsilon} \mathrm{e}^{-\frac{\alpha t}{\varepsilon}}+\frac{1}{\varepsilon} c_{1} M_{1}^{*} \int_{0}^{t} \mathrm{e}^{-\frac{\alpha(t-\tau)}{\varepsilon}} \mathrm{d} \tau+\frac{1}{\varepsilon}\|F\|_{\infty} \int_{0}^{t} \mathrm{e}^{-\frac{\alpha(t-\tau)}{\varepsilon}} \mathrm{d} \tau+\frac{1}{\varepsilon} c_{0} M_{1} \int_{0}^{t} \mathrm{e}^{-\frac{\alpha(t-\tau)}{\varepsilon}} \mathrm{d} \tau,
$$

which proves (6) for $k=1$. To obtain (6) for $k=2$, first from Equation (1) we have

$$
\left|u^{\prime \prime}(0)\right| \leq \frac{\left|a(0) u^{\prime}(0)-f(0, u(0), \lambda)\right|}{\varepsilon},
$$

from which after taking into consideration here $u^{\prime}(0)=\frac{\mu_{1}}{\varepsilon}$ and (4)

$$
\left|u^{\prime \prime}(0)\right| \leq \frac{C}{\varepsilon^{2}} .
$$

Next, differentiation (1) gives

$$
\begin{gathered}
\varepsilon v^{\prime \prime}(t)+a(t) v^{\prime}(t)+\varphi(t)=0, \quad 0<t<T, \\
v^{\prime}(0)=0\left(\varepsilon^{-2}\right)
\end{gathered}
$$

with

$$
\begin{gathered}
v(t)=u^{\prime}(t), \\
\varphi(t)=a^{\prime}(t) v(t)-\frac{\partial f}{\partial t}(t, u(t), \lambda)-\frac{\partial f}{\partial u}(t, u(t), \lambda),
\end{gathered}
$$

and due to our assumptions clearly,

$$
|\varphi(t)| \leq C\left(1+\frac{1}{\varepsilon^{k}} \mathrm{e}^{-\frac{\alpha t}{\varepsilon}}\right)
$$

Consequently, from (17), (18) we have

$$
\begin{aligned}
\left|v^{\prime}(t)\right| & \leq \frac{C}{\varepsilon^{2}} \mathrm{e}^{-\frac{\alpha t}{\varepsilon}}+\frac{1}{\varepsilon} \int_{0}^{t}|\varphi(\tau)| \mathrm{e}^{-\frac{\alpha(t-\tau)}{\varepsilon}} \mathrm{d} \tau \\
& =\frac{C}{\varepsilon^{2}} \mathrm{e}^{-\frac{\alpha t}{\varepsilon}}+C\left(1+\frac{1}{\varepsilon^{2}} \mathrm{e}^{-\frac{\alpha t}{\varepsilon}} \int_{0}^{t} \mathrm{e}^{-\frac{\alpha(t-\tau)}{\varepsilon}} \mathrm{d} \tau\right. \\
& =\frac{C}{\varepsilon^{2}} \mathrm{e}^{-\frac{\alpha t}{\varepsilon}}+C\left(1+\frac{1}{\varepsilon^{2}} \mathrm{e}^{-\frac{\alpha t}{\varepsilon}}\right)
\end{aligned}
$$

which proves (6) for $k=2$.

Example. Consider the particular problem

$$
\varepsilon u^{\prime \prime}(t)+2 u^{\prime}(t)+\lambda+\tanh (t+\lambda)=f(t), 0<t<1,
$$




$$
u(0)=1, u^{\prime}(0)=\frac{\mu_{1}}{\varepsilon}, u(1)=0,
$$

where, $\mu_{1}$ and $f(t)$ selected so that the solution is

$$
u(t)=\gamma_{1}+\gamma_{2} \mathrm{e}^{-\frac{2}{\varepsilon} t}+\frac{1}{2+\varepsilon} \mathrm{e}^{t}, \quad \lambda=0.5
$$

with

$$
\gamma_{1}=-\frac{\mathrm{e}+(1+\varepsilon) \mathrm{e}^{-\frac{2}{\varepsilon}}}{(2+\varepsilon)\left(1-\mathrm{e}^{-\frac{2}{\varepsilon}}\right)}, \quad \gamma_{2}=\frac{1+\varepsilon+\mathrm{e}}{(2+\varepsilon)\left(1-\mathrm{e}^{-\frac{2}{\varepsilon}}\right)} .
$$

First and second derivatives have the form

$$
u^{(k)}(t)=\left(-\frac{2}{\varepsilon}\right)^{k} \gamma_{2} \mathrm{e}^{-\frac{2}{\varepsilon} t}+\frac{1}{2+\varepsilon} \mathrm{e}^{t}, k=1,2 .
$$

Therefore we observe here the accordance in our theoretical results described above.

\section{References}

[1] Pomentale, T. (1976) A Constructive Theorem of Existence and Uniqueness for the Problem. Zeitschrift für Angewandte Mathematik und Mechanik, 56, 387-388. http://dx.doi.org/10.1002/zamm.19760560806

[2] Goma, I.A. (1977) Method of Successive Approximations in a Two-Point Boundary Problem with Parameter. Ukrainian Mathematical Journal, 29, 594-599. http://dx.doi.org/10.1007/BF01085968

[3] Jankowski, T. (2001) Monotone İterations for Differential Problems. Miskolc Mathematical Notes, 2, 31-38.

[4] Jankowski, T. and Lakshmikantham, V. (1997) Monotone Iterations for Differential Equations with a Parameter. Journal of Applied Mathematics and Stochastic Analysis, 10, 273-278. http://dx.doi.org/10.1155/S1048953397000348

[5] Jankowski, T. (1999) Generalization of the Method of Quasilinearization for Differential Problems with a Parameter, Dynamic Systems an Applications, 8, 53-72.

[6] Rontó, M. and Csikos-Marinets, T. (2000) On the Investigation of Some Non-Linear Boundary Value Problems with Parameters. Miskolc Mathematical Notes, 1, 157-166.

[7] Rontó, M. (2000) On Non-Linear Boundary Value Problems Containing Parameters. Archivum Mathematicum, 36, 585-593.

[8] Staněk, S. (1997) Nonlinear Boundary Value Problem for Second Order Differential Equations Depending on a Parameter. Mathematica Slovaca, 47, 439-449.

[9] Zhang, P. (2011) Existence of Positive Solutions for Nonlocal Second-Order Boundary Value Problem with Variable Parameter in Banach Spaces. Fixed Point Theory and Applications, 43, 1687-1812. http://dx.doi.org/10.1186/1687-1812-2011-43

[10] Fěckan, M. (1994) Parametrized Singularly Perturbed Boundary Value Problems. Journal of Mathematical Analysis and Applications, 188, 426-435. http://dx.doi.org/10.1006/jmaa.1994.1436

[11] Amiraliyev, G.M., Kudu M. and Duru, H. (2004) Finıte-Difference Method for Parameterized Singularly Pertur Bed Problem. Journal of Applied Mathematics, 3, 191-199. http://dx.doi.org/10.1155/S1110757X0440103X

[12] Amiraliyev, G.M., Kudu M. and Duru, H. (2006) Uniform Difference Method for a Parameterized Singular Pertur Bation Problem. Applied Mathematics and Computation, 175, 89-100. http://dx.doi.org/10.1016/j.amc.2005.07.068

[13] Amiraliyeva I.G. and Amiraliyev, G.M. (2009) Uniform Difference Method for Parameterized Singularly Pertur Bed Delay Differential Equations. Numerical Algorithms, 52, 509-552. http://dx.doi.org/10.1007/s11075-009-9295-y

[14] Turkyilmazoglu, M. (2011) Analytic Approximate Solutions of Parameterized Unperturbed and Singularly Perturbed Boundary Value Problems. Applied Mathematical Modelling, 35, 3879-3886. http://dx.doi.org/10.1016/j.apm.2011.02.011

[15] Nayfeh, A.H. (1981) Introduction to Perturbation Techniques. JohnWiley \& Sons, New York.

[16] O’Malley Jr., R.E. (1991) Singular Perturbation Methods for Ordinary Differential Equations. Springer-Verlag, New York. http://dx.doi.org/10.1007/978-1-4612-0977-5 
Scientific Research Publishing (SCIRP) is one of the largest Open Access journal publishers. It is currently publishing more than 200 open access, online, peer-reviewed journals covering a wide range of academic disciplines. SCIRP serves the worldwide academic communities and contributes to the progress and application of science with its publication.

Other selected journals from SCIRP are listed as below. Submit your manuscript to us via either submit@scirp.org or Online Submission Portal.
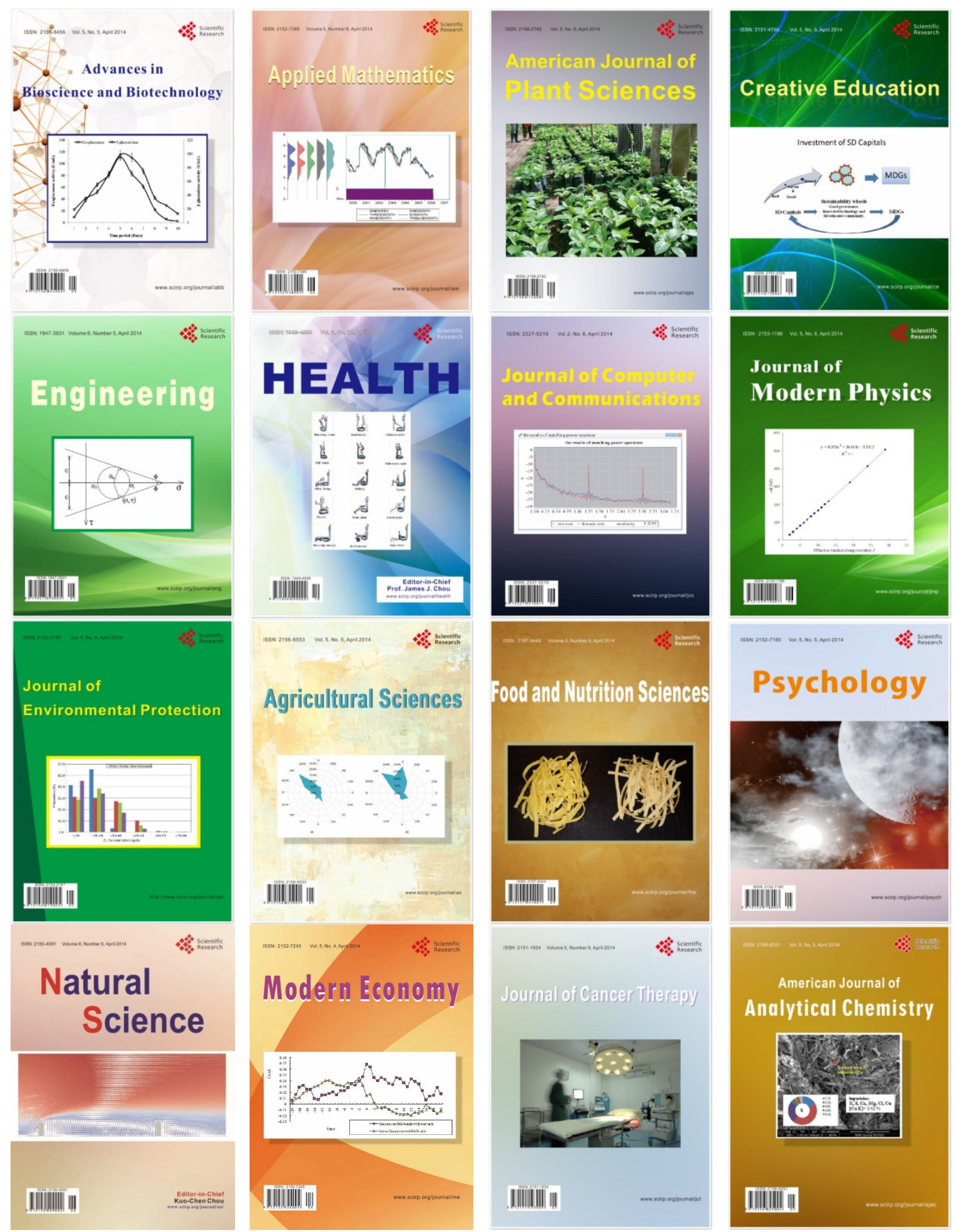